\title{
FORÇA MUSCULAR RESPIRATÓRIA, POSTURA CORPORAL, INTENSIDADE VOCAL E TEMPOS MÁXIMOS DE FONAÇÃO NA DOENÇA DE PARKINSON
}

\author{
Respiratory muscle strength, body posture, vocal intensity \\ and maximum phonation times in Parkinson Disease
}

\author{
Fernanda Vargas Ferreira ${ }^{(1)}$, Carla Aparecida Cielo ${ }^{(2)}$, Maria Elaine Trevisan ${ }^{(3)}$
}

\begin{abstract}
RESUMO
Tema: verificar os achados de força muscular respiratória (FMR), postura corporal (PC), intensidade vocal (IV) e tempos máximos de fonação (TMF), em indivíduos com Doença de Parkinson (DP) e casos de controle, conforme o sexo, o estágio da DP e o nível de atividade física (AF). Procedimentos: três homens e duas mulheres com DP, entre 36 e 63 anos (casos de estudo - CE), e cinco indivíduos sem doenças neurológicas, pareados em idade, sexo e nível de AF (casos de controle - CC). Avaliadas a FMR, PC, IV e TMF. Resultados: homens: diminuição mais acentuada dos TMF, IV e FMR nos parkinsonianos, mais alterações posturais nos idosos; mulheres com e sem DP: alterações posturais similares, relação positiva entre estágio, nível de AF e as demais medidas. Conclusões: verificou-se nas parkinsonianas, prejuízo na IV e nos parkinsonianos déficits nos TMF, IV e FMR. Sugerem-se novos estudos sob um viés interdisciplinar.
\end{abstract}

DESCRITORES: Doença de Parkinson; Respiração; Voz; Postura

\section{INTRODUÇÃO}

A Doença de Parkinson (DP) é uma enfermidade neurodegenerativa, decorrente de lesão nos gânglios basais. Ocorre a partir dos 50 anos, em ambos os sexos, com repercussões nos sistemas respiratório, músculoesquelético e estomatognático ${ }^{1-3}$.

Há redução da capacidade vital e da força muscular respiratória, possivelmente pela rigidez

(1) Fisioterapeuta; Professora Assistente do Curso de Fisioterapia do Centro Universitário Franciscano/RS; Mestre em Distúrbios da Comunicação Humana pela Universidade Federal de Santa Maria/RS, Brasil.

(2) Fonoaudióloga; Professora Adjunta do Departamento de Fonoaudiologia da Universidade Federal de Santa Maria/ RS; Doutora em Lingüística Aplicada pela Pontifícia Universidade Católica do Rio Grande do Sul/RS, Brasil.

(3) Fisioterapeuta; Professora Assistente do Departamento de Fisioterapia e Reabilitação da Universidade Federal de Santa Maria/RS; Mestre em Ciência do Movimento Humano - Área de Fisiologia do Exercício pela Universidade Federal de Santa Maria/RS, Brasil.

Conflito de interesses: inexistente muscular e alterações na coluna vertebral, podendo gerar déficit restritivo na ventilação ${ }^{2-6}$. A postura do parkinsoniano é a de flexão da cabeça, hipercifose torácica, protração de ombros e flexão dos braços $^{3,4}$. Os desvios posturais de cabeça limitam os movimentos da mandíbula, interferindo na $\mathrm{voz}^{6,7}$, já que existem relações anatomofuncionais do sistema estomatognático com a região cérvico-escapular ${ }^{3,6,7}$. Destacam-se, na disartrofonia hipocinética, monotonia de intensidade ${ }^{2,7,8}$; disprosódia $^{8-10}$; disfluência ${ }^{9-11}$ e alteração da velocidade de fala $^{2,8,10,11}$.

A redução da intensidade vocal tende a tornar a comunicação verbal ininteligível $\left.\right|^{2,7,9,11}$, sendo que o padrão hipercifótico, frequente na $\mathrm{DP}$, resulta em menor expansão do gradil costal ${ }^{4,5}$ e em menores volumes pulmonares ${ }^{1,3,5}$. Ainda, a hipofonia apresenta possível etiologia baseada em alterações na configuração das pregas vocais ${ }^{2,8,11}$.

Consequentemente, os tempos máximos de fonação (TMF) podem apresentar-se reduzidos ${ }^{10,11}$, indicando a alteração da dinâmica respiratória e/ou da coaptação das pregas vocais, com desequilíbrio 
das forças aerodinâmicas respiratórias e mioelásticas da laringe ${ }^{11,12}$. Fisiologicamente, a IV e os TMF diminuem com o avanço da idade devido a redução do tônus muscular, fraqueza e atrofia da musculatura laríngea e ao decréscimo da força muscular respiratória $(\mathrm{FMR})^{5,12,13}$.

Acerca da possível relação entre fonação, postura e respiração na $\mathrm{DP}$, esta pesquisa teve como objetivo verificar FMR, PC, IV e TMF, conforme o sexo, estágio da DP e o nível de atividade física.

\section{APRESENTAÇÃO DO CASO}

A população da qual foram selecionados os casos a serem estudados compreendeu indivíduos com diagnóstico neurológico de DP, ambos os sexos, entre 35 e 65 anos, falantes do português brasileiro (PB). Os casos de controle foram selecionados a partir de uma população de indivíduos sem DP, ambos os sexos, falantes do PB, pareados de acordo com a idade, o sexo e o nível de atividade física.

Os critérios de exclusão para os casos de estudo (CE) e de controle (CC) foram: déficit cognitivo; avaliação laringológica com anormalidades, que não as alterações laríngeas típicas da DP e ou do envelhecimento; sintomas ou sinais espirométricos de doença pulmonar obstrutiva crônica; estar realizando tratamento fonoaudiológico e/ou otorrinolaringológico; ser profissional da voz; ser tabagista e/ou consumir álcool em excesso; perda auditiva; cantar em coros.

Todos os indivíduos foram submetidos à entrevista semi-estruturada, avaliação otorrinolaringológica, espirométrica e auditiva, por profissionais especialistas. A partir dos resultados dessas avaliações, foram selecionados para o estudo apenas os sujeitos que atenderam aos critérios mencionados.

Para o pareamento quanto ao nível de atividade física, todos os sujeitos responderam ao Questionário Internacional de Atividade Física (IPAQ) ${ }^{14}$. Dos oito indivíduos que se apresentaram como voluntários, excluíram-se três por perda auditiva.

Assim, constituiu-se um grupo de CE com três homens e duas mulheres, entre 36 e 63 anos, e um grupo de CC constituído por cinco indivíduos sem doenças neurológicas. Esses sujeitos, pareados em CE e CC, nas idades de 36, 56, 57, 61 e 63 anos, cada par respectivamente, foram submetidos às avaliações para a coleta de dados.

Para os CE, realizou-se a delimitação dos estágios, por meio da Escala de Hohen e Yahr Modificada $^{15}$.

A avaliação postural foi realizada por uma fisioterapeuta sem conhecimentos sobre os propósitos da pesquisa. O sujeito usou traje de banho, em bipedestação, avaliado nas posições ântero-posterior, póstero-anterior e em perfil. Considerou-se a posição cérvico-escapular e as curvaturas da coluna vertebral ${ }^{16}$.

A intensidade vocal foi realizada por fonoaudióloga, por meio de um decibelímetro digital (marca Homis, modelo 413) posicionado a quatro centímetros da boca do sujeito, em sala acusticamente tratada. Solicitou-se ao sujeito, em bipedestação, que após inspiração profunda, emitisse a vogal /a/ sustentada pelo tempo mais prolongado possível ${ }^{17}$. Adotaram-se os valores correspondentes à média para indivíduos adultos do sexo feminino de $63,75 \mathrm{~dB}$ e de $63,01 \mathrm{~dB}$ para o sexo masculino ${ }^{18} \mathrm{e}$, para idosos, de $68,9 \mathrm{~dB}^{19}$.

As mensurações da Pressão inspiratória máxima (Pimáx) e Pressão expiratória máxima (Pemáx) foram feitas por meio de um manovacuômetro digital (marca Microhard MVD 500). Os indivíduos em bipedestação, tendo as narinas ocluídas com clipe nasal, foram instruídos a exalar até o volume residual (VR) ou inalar até a capacidade pulmonar total (CPT) antes de serem estimulados a inalar/exalar com esforço máximo para mensurar a Pimáx e Pemáx respectivamente. Realizaram-se de três a cinco manobras máximas, com intervalo de descanso de aproximadamente um minuto, sendo registrado o valor mais alto. Adotaram-se os valores referenciais da literatura ${ }^{20}$.

Os TMF foram coletados por fonoaudióloga, que solicitou ao indivíduo, após uma inspiração máxima, que sustentasse durante toda uma expiração, em tom e intensidade habituais, as vogais, o /e/ áfono e as consoantes /s/, /z/, que foram gravadas digitalmente (marca CABO 4P BK-USB22 "BAK") com o microfone posicionado a quatro centímetros da boca do sujeito ${ }^{17,21}$ e também cronometradas em segundos (cronômetro digital Technos) para a quantificação do TMF de cada um. Cada fonema foi sustentado por três vezes, sendo escolhido o maior valor em segundos de cada fonema ${ }^{21}$. Após a coleta dos TMF, foram realizadas as relações s/z, e e áfono/e. Consideraram-se os TMF para adultos do sexo feminino acima de 15 segundos e para o sexo masculino acima de 20 segundos $^{21}$. Para idosos do sexo feminino, entre 10 e 15 segundos e do sexo masculino, entre 15 e 20 segundos $^{12}$. Consideraram-se os valores da relação $\mathrm{s} / \mathrm{z}$ para adultos de 0,99 a 1,2 e para idosos de 0,76 a 0,82 22 e para a relação e áfono/e de 0,8 a 1,2 ${ }^{17}$, tanto para adultos como para idosos.

Todas as avaliações foram realizadas após a medicação anti-parkinsoniana (período ON).

Os participantes assinaram o Termo de Consentimento Livre e Esclarecido (Resolução 196/96 - 
CNS), sendo o presente trabalho previamente aprovado pelo Comitê de Ética em Pesquisa da instituição de origem (n 091/2005).

A análise dos dados coletados foi realizada de forma descritiva, observando-se as tendências e possíveis relações apresentadas pelos CE e CC, pareados segundo sexo, idade e nível de atividade física, nas avaliações da FMR, PC, IV e TMF.

\section{RESULTADOS}

O grupo de CE foi constituído de cinco indivíduos, três homens e duas mulheres, na faixa etária entre 36 e 63 anos, classificados na Escala de Hohen e Yahr Modificada ${ }^{15}$, sendo um indivíduo em cada estágio (1,5; 2 e 3) e dois indivíduos no estágio 2,5; pareados segundo idade, sexo e nível de atividade física com cinco indivíduos sem doenças neurológicas (CC), todos submetidos à avaliação FMR, da PC, da IV e dos TMF.
Par a par, observaram-se as seguintes características, como segue.

Caso de Estudo 1 (CE1), sexo feminino, 57 anos, estágio 2,5, irregularmente ativo $\mathrm{A}$, pareada com Caso de Controle 1 (CC1), sexo feminino, 57 anos, irregularmente ativo A (Figura 1).

Caso de Estudo 2 (CE2), sexo feminino, 56 anos, estágio 1,5, ativa, pareada com Caso de Controle 2 (CC2), sexo feminino, 56 anos, ativa (Figura 2).

Caso de Estudo 3 (CE 3), sexo masculino, 63 anos, estágio 2,5, muito ativo, pareado com Caso de Controle 3, (CC3), sexo masculino, 63 anos, muito ativo (Figura 3 ).

Caso de Estudo 4 (CE4), sexo masculino, 59 anos, estágio 3, sedentário, pareado com Caso de Controle 4, (CC4), sexo masculino, 59 anos, sedentário (Figura 4).

Caso de Estudo 5 (CE5), sexo masculino, 36 anos, estágio 2, ativo, pareado com Caso de Controle 5, (CC5), sexo masculino, 36 anos, ativo (Figura 5).

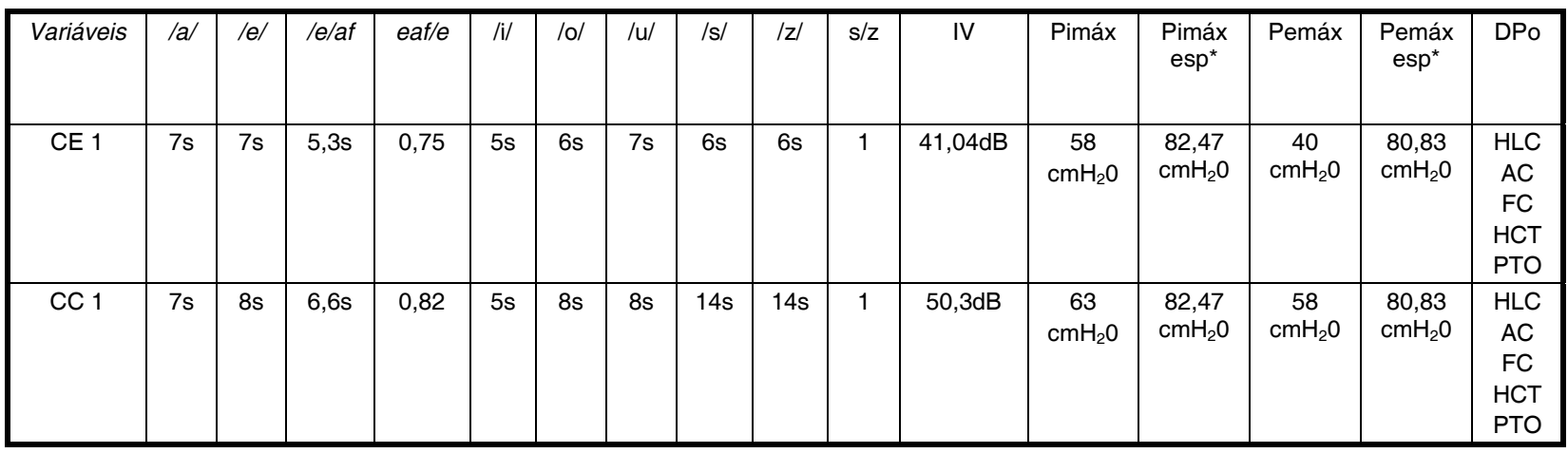

Legenda: /e/af = /e/ áfono; IV = intensidade vocal; Pimáx = pressão inspiratória máxima; Pimáxesp = pressão inspiratória máxima esperada; Pemáx = pressão expiratória máxima; Pemáxesp = pressão expiratória máxima esperada; $\mathrm{DPo}=$ desvios posturais; $\mathrm{HLC}=$ hiperlordose cervical; $\mathrm{AC}$ = anteriorização da cabeça; $\mathrm{FC}=$ flexão da cabeça; $\mathrm{HCT}$ = hipercifose torácica; $\mathrm{PTO}=$ protração de ombros

\section{Figura 1 - Características do Caso de Estudo 1 e Caso de Controle 1}

\begin{tabular}{|c|c|c|c|c|c|c|c|c|c|c|c|c|c|c|c|c|}
\hline Variáveis & la/ & le/ & le/af & eaf/e & /i/ & $10 /$ & $/ \mathrm{u} /$ & $/ \mathrm{s} /$ & $|z|$ & $s / z$ & IV & Pimáx & $\begin{array}{c}\text { Pimáx } \\
\text { esp }^{*}\end{array}$ & Pemáx & $\begin{array}{c}\text { Pemáx } \\
\text { esp }^{\star}\end{array}$ & DPo \\
\hline CE 2 & $10 \mathrm{~s}$ & 9s & $7,2 \mathrm{~s}$ & 0,8 & 9s & $10 \mathrm{~s}$ & $10 \mathrm{~s}$ & $10 \mathrm{~s}$ & $9 s$ & 1,1 & $\begin{array}{c}48,7 \\
d B\end{array}$ & $\begin{array}{c}41 \\
\mathrm{cmH}_{2} \mathrm{O}\end{array}$ & $\begin{array}{c}82,96 \\
\mathrm{cmH}_{2} \mathrm{O}\end{array}$ & $\begin{array}{c}55 \\
\mathrm{cmH} 20\end{array}$ & $\begin{array}{c}81,44 \\
\mathrm{cmH}_{2} \mathrm{O}\end{array}$ & $\begin{array}{c}\text { HLC } \\
\text { AC } \\
\text { FC } \\
\text { HCT } \\
\text { PTO }\end{array}$ \\
\hline CC 2 & $7 \mathrm{~s}$ & $7,3 \mathrm{~s}$ & $5,7 \mathrm{~s}$ & 0,78 & $10 \mathrm{~s}$ & $11 \mathrm{~s}$ & $10 \mathrm{~s}$ & $13 \mathrm{~s}$ & $13 \mathrm{~s}$ & 1 & $\begin{array}{c}50,9 \\
\mathrm{~dB}\end{array}$ & $\begin{array}{c}71 \\
\mathrm{cmH}_{2} \mathrm{O}\end{array}$ & $\begin{array}{c}82,96 \\
\mathrm{cmH}_{2} \mathrm{O}\end{array}$ & $\begin{array}{c}51 \\
\mathrm{cmH}_{2} \mathrm{O}\end{array}$ & $\begin{array}{l}81,44 \\
\mathrm{cmH}_{2} \mathrm{O}\end{array}$ & $\begin{array}{c}\mathrm{HLC} \\
\mathrm{AC} \\
\mathrm{FC} \\
\mathrm{HCT}\end{array}$ \\
\hline
\end{tabular}

Legenda: /e/af = /e/ áfono; IV = intensidade vocal; Pimáx = pressão inspiratória máxima; Pimáxesp = pressão inspiratória máxima esperada; Pemáx = pressão expiratória máxima; Pemáxesp = pressão expiratória máxima esperada; $\mathrm{DPo}=$ desvios posturais; $\mathrm{HLC}=$ hiperlordose cervical; $\mathrm{AC}$ = anteriorização da cabeça; FC = flexão da cabeça; $\mathrm{HCT}$ = hipercifose torácica; $\mathrm{PTO}=$ protração de ombros 


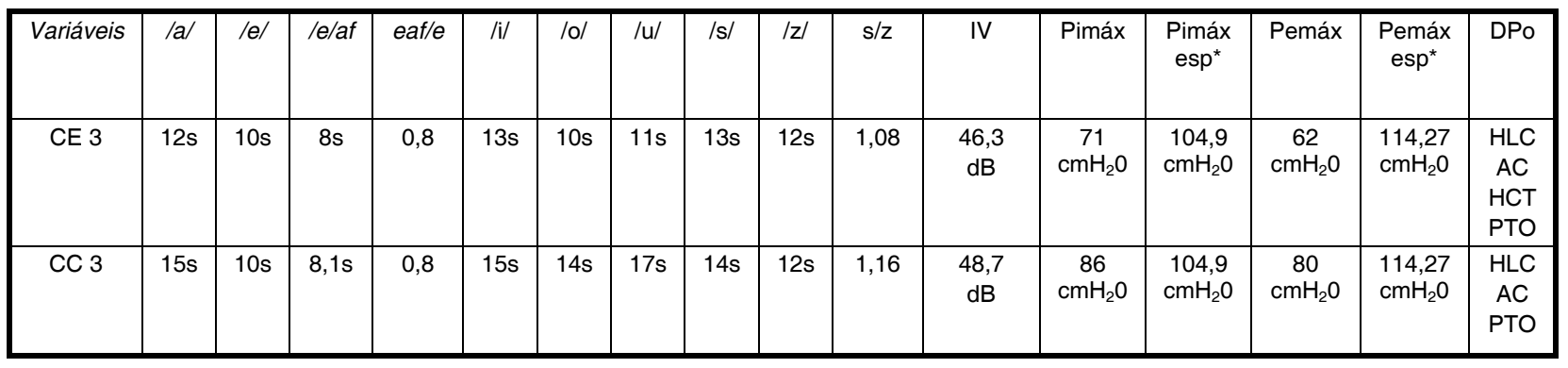

Legenda: /e/af = /e/ áfono; IV = intensidade vocal; Pimáx = pressão inspiratória máxima; Pimáxesp = pressão inspiratória máxima esperada; Pemáx = pressão expiratória máxima; Pemáxesp = pressão expiratória máxima esperada; $D P o=$ desvios posturais; HLC = hiperlordose cervical; $\mathrm{AC}$ = anteriorização da cabeça; $\mathrm{FC}=$ flexão da cabeça; $\mathrm{HCT}$ = hipercifose torácica; $\mathrm{PTO}=$ protração de ombros

Figura 3 - Características do Caso de Estudo 3 e Caso de Controle 3

\begin{tabular}{|c|c|c|c|c|c|c|c|c|c|c|c|c|c|c|c|c|}
\hline Variáveis & $\mid a /$ & le/ & le/af & eaf/e & /i/ & $10 /$ & $/ u /$ & $/ \mathrm{s} /$ & $|z|$ & $s / z$ & IV & Pimáx & $\begin{array}{c}\text { Pimáx } \\
\text { esp* }\end{array}$ & Pemáx & $\begin{array}{c}\text { Pemáx } \\
\text { esp }\end{array}$ & DPo \\
\hline CE 4 & $5 s$ & $5,1 \mathrm{~s}$ & $5,4 \mathrm{~s}$ & 1,05 & $5 s$ & $5 s$ & $4 s$ & $6 s$ & $5 s$ & 1,2 & $\begin{array}{l}29 \\
d B\end{array}$ & $\begin{array}{c}29 \\
\mathrm{cmH}_{2} \mathrm{O}\end{array}$ & $\begin{array}{c}108,1 \\
\mathrm{cmH}_{2} \mathrm{O}\end{array}$ & $\begin{array}{c}39 \\
\mathrm{cmH}_{2} \mathrm{O}\end{array}$ & $\begin{array}{l}117,51 \\
\mathrm{cmH}_{2} \mathrm{O}\end{array}$ & $\begin{array}{c}\text { HLC } \\
\text { AC } \\
\text { HCT } \\
\text { PTO }\end{array}$ \\
\hline CC 4 & $17 \mathrm{~s}$ & $11 \mathrm{~s}$ & $6,3 \mathrm{~s}$ & 0,57 & $12 \mathrm{~s}$ & $12 \mathrm{~s}$ & $14 \mathrm{~s}$ & $9 \mathrm{~s}$ & $8 \mathrm{~s}$ & 1,12 & $\begin{array}{c}63,8 \\
d B\end{array}$ & $\begin{array}{c}107 \\
\mathrm{cmH}_{2} \mathrm{O}\end{array}$ & $\begin{array}{c}108,1 \\
\mathrm{cmH}_{2} \mathrm{O}\end{array}$ & $\begin{array}{c}85 \\
\mathrm{cmH}_{2} \mathrm{O}\end{array}$ & $\begin{array}{l}117,51 \\
\mathrm{cmH}_{2} 0\end{array}$ & $\begin{array}{c}\text { HLC } \\
\text { AC } \\
\text { PTO }\end{array}$ \\
\hline
\end{tabular}

Legenda: /e/af = /e/ áfono; IV = intensidade vocal; Pimáx = pressão inspiratória máxima; Pimáxesp = pressão inspiratória máxima esperada; Pemáx = pressão expiratória máxima; Pemáxesp = pressão expiratória máxima esperada; $\mathrm{DPo}=$ desvios posturais; $\mathrm{HLC}=$ hiperlordose cervical; $\mathrm{AC}$ = anteriorização da cabeça; $\mathrm{FC}$ = flexão da cabeça; HCT = hipercifose torácica; $\mathrm{PTO}=$ protração de ombros

\section{Figura 4 - Características do Caso de Estudo 4 e Caso de Controle 4}

\begin{tabular}{|c|c|c|c|c|c|c|c|c|c|c|c|c|c|c|c|c|}
\hline Variáveis & lal & le/ & le/af & eaf/e & /i/ & /0/ & $/ \mathrm{u} /$ & $/ \mathrm{s} /$ & $|z|$ & $s / z$ & IV & Pimáx & $\begin{array}{c}\text { Pimáx } \\
\text { esp }^{*}\end{array}$ & Pemáx & $\begin{array}{c}\text { Pemáx } \\
\text { esp* }^{*}\end{array}$ & DPo \\
\hline CE 5 & $8 s$ & $7 \mathrm{~s}$ & $4,8 \mathrm{~s}$ & 0,68 & $5 s$ & $7 \mathrm{~s}$ & $5 s$ & $6 s$ & $5 s$ & 1,12 & $\begin{array}{c}53,8 \\
d B\end{array}$ & $\begin{array}{c}120 \\
\mathrm{cmH}_{2} \mathrm{O}\end{array}$ & $\begin{array}{c}126,5 \\
\mathrm{cmH}_{2} \mathrm{O}\end{array}$ & $\begin{array}{c}102 \\
\mathrm{cmH}_{2} \mathrm{O}\end{array}$ & $\begin{array}{l}136,14 \\
\mathrm{cmH}_{2} \mathrm{O}\end{array}$ & $\begin{array}{c}\text { HLC } \\
\text { AC } \\
\text { FC }\end{array}$ \\
\hline CC 5 & $11 \mathrm{~s}$ & $10 \mathrm{~s}$ & $7,6 s$ & 0,76 & $12 \mathrm{~s}$ & $13 \mathrm{~s}$ & $13 \mathrm{~s}$ & $11 \mathrm{~s}$ & $12 \mathrm{~s}$ & 0,91 & $\begin{array}{c}59,2 \\
d B\end{array}$ & $\begin{array}{c}109 \\
\mathrm{cmH}_{2} \mathrm{O}\end{array}$ & $\begin{array}{c}126,5 \\
\mathrm{cmH}_{2} \mathrm{O}\end{array}$ & $\begin{array}{c}107 \\
\mathrm{cmH}_{2} \mathrm{O}\end{array}$ & $\begin{array}{l}136,14 \\
\mathrm{cmH}_{2} \mathrm{O}\end{array}$ & $\begin{array}{c}\text { HLC } \\
\text { AC } \\
\text { FC } \\
\text { HCT }\end{array}$ \\
\hline
\end{tabular}

Legenda: /e/af = /e/ áfono; IV = intensidade vocal; Pimáx = pressão inspiratória máxima; Pimáxesp = pressão inspiratória máxima esperada; Pemáx = pressão expiratória máxima; Pemáxesp = pressão expiratória máxima esperada; $\mathrm{DPo}=$ desvios posturais; HLC = hiperlordose cervical; $\mathrm{AC}$ = anteriorização da cabeça; $\mathrm{FC}=$ flexão da cabeça; HCT = hipercifose torácica; $\mathrm{PTO}=$ protração de ombros

\section{Figura 5 - Características do Caso de Estudo 5 e Caso de Controle 5}

* Em cada par de casos, os valores correspondentes às pressões respiratórias máximas esperadas foram apresentados nos respectivos quadros, já que, os resultados variaram de acordo com o sexo e a idade, conforme a literatura ${ }^{20}$.

\section{DISCUSSÃO}

Neste estudo, três indivíduos eram do sexo masculino e dois do sexo feminino, dos quais quatro apresentaram sinais e sintomas em torno da quinta década de vida, em concordância com estudos que apontam discreta predominância nos homens, a partir dos 50 anos $^{1,3,11}$.

Quanto aos estágios da DP, os dados evidenciaram que a maioria dos indivíduos encontrou-se nos estágios intermediários, convergindo com outros estudos ${ }^{11,23}$. 
Apenas um sujeito foi classificado como sedentário, possivelmente por estar no estágio de DP mais avançado, concordando com a afirmação de que à medida que a doença evolui, os parkinsonianos se exercitam com menor freqüência ${ }^{1,3-5}$.

Ao se verificar a relação entre os pares de CE e CC no sexo feminino, os resultados dos TMF, das Pimáx e Pemáx e das relações e áfono/e e s/z apresentaram-se similares ou próximos. Possivelmente, a melhor performance nas avaliações decorre da associação entre os menores estágios da DP e o maior nível de atividade física.

Como o envelhecimento gera alterações como perda de massa muscular, menor expansão do gradil costal e encurtamento do diafragma, com conseqüente prejuízo nas capacidades respiratórias $^{3,20}$, seria esperada uma repercussão negativa no desempenho desses sujeitos.

Uma possível justificativa, além dos estágios iniciais da DP nas parkinsonianas, procede também da atividade física que incrementa a FMR pelo estímulo à flexibilidade do diafragma. Os exercícios físicos aumentam o tônus dos abdominais que servem de apoio ao deslocamento céfalo-caudal do diafragma ${ }^{4,5}$. A atividade física tende a otimizar as funções cardiorrespiratórias, refletindo-se no fluxo expiratório ${ }^{4,5,24}$.

A relação e áfono/e, semelhante à relação $\mathrm{s} / \mathrm{z}$, evidencia a coordenação entre o sopro expiratório e as pregas vocais durante a fonação, e a sustentação da vogal áfona /e/ constitui um indicador clínico do controle da saída do ar, exclusivamente por meio do suporte respiratório, ocorrendo o mesmo com a sustentação do fonema /s/. Na emissão sustentada de /z/ e de /e/, observa-se o papel da fonte glótica no controle do ar expiratório à fonação ${ }^{17,21}$.

Notou-se que as parkinsonianas e os seus CC exibiram a relação $\mathrm{s} / \mathrm{z}$ dentro do esperado ${ }^{12,22}$, contrário à literatura, já que o envelhecimento e a DP tendem a prejudicar a FMR e TMF, conseqüência da incoordenação neuromuscular presente nos sistemas fonatório, articulatório e respiratório $^{2,7,10-13}$. Uma possível justificativa para esse achado é o controle do fluxo expiratório, conforme as performances nas Pimáx e Pemáx, pois presumivelmente houve substrato respiratório. Além disso, os TMF isolados de /s/ e de /z/ apresentam o controle articulatório como um fator mascarante; e o fato de estarem rebaixados nas parkinsonianas, resultam numa relação matemática em torno de 1 , o que não significa equilíbrio entre o aporte respiratório e o funcionamento glótico à fonação, e sim, um viés matemático da medida.

Porém, na relação e áfono/e, tanto nas parkinsonianas quanto nos seus CC, verificou-se alteração com valores abaixo ou próximos do limiar inferior ${ }^{17}$, possivelmente por hipercinesia compensatória a partir da falta de resistência glótica no caso da DP e por comportamento vocal incorreto no caso dos $\mathrm{CC}^{17,21}$.

A IV depende da interação periférica entre a pressão de ar subglótica, a quantidade de fluxo aéreo, a resistência glótica e as mudanças na configuração do trato vocal $7,10,12,17$. Neste estudo, a IV das parkinsonianas, independente do estágio da doença, apresentou-se em piores condições quando comparadas aos $\mathrm{CC}$, que também se encontraram inferiores à literatura ${ }^{22}$. Provavelmente, essa medida mostrou-se mais prejudicada nas parkinsonianas do que nas idosas pela rigidez dos músculos respiratórios, visto que a expansão da caixa torácica e os volumes pulmonares são a base respiratória para a IV ${ }^{1,4,5,14,25}$. Também é possível afirmar que a musculatura laríngea apresenta-se afetada na DP, sendo que algumas pesquisas sugerem hiperexcitabilidade dos músculos tireoaritenóideo e cricotireóideo ${ }^{8,9}$. Outras apontam redução nos potenciais de ação ${ }^{1,21}$, sendo esperada a diminuição da IV, comum no discurso do parkinsoniano $0^{2,9,11,13}$.

No entanto, as medidas de Pimáx e Pemáx, bem como algumas medidas de TMF e a relação s/z mostraram-se próximas aos valores esperados para idosas $5,12,13,21,25$, mesmo na presença da $\mathrm{DP}^{2,9,21}$. Assim, sugere-se que as idosas, com e sem DP, ainda evidenciaram coordenação pneumofonoarticulatória.

É necessário considerar que as alterações funcionais ocorrem em maior número, freqüência e gravidade de acordo com a evolução da DP,15,24 e ponderar que os fenômenos da voz variam em complexidade e extensão ${ }^{8-10}$, podendo ocorrer nas fases iniciais ${ }^{2,11}$, podendo justificar a diminuição da IV sem prejuízo evidente de TMF e da relação s/z, como neste estudo.

Quanto à $P C$, não houve substanciais diferenças entre os $\mathrm{CE}$ e CC femininos, pois ocorreu um encurtamento seletivo dos músculos flexores, adutores e pronadores, gerando a postura em flexão, tanto no envelhecimento (CC) quanto na DP (CE) $)^{4,16,24}$.

Quanto ao sexo masculino, os parkinsonianos, em geral, exibiram resultados inferiores nos TMF, na IV e na FMR em relação aos CC, aparentemente sem relação com a idade, o nível de atividade física e o estágio da DP. Possivelmente, pelos efeitos do envelhecimento no aparelho fonador e no sistema respiratório ${ }^{5,12,13,25}$, acentuados pelas limitações da $\mathrm{DP}^{2,9,21}$.

Com base nesses achados, pode-se supor a deterioração da inter-relação entre as forças expiratórias e mioelásticas da laringe, uma vez que os decréscimos dos TMF e da IV indicaram a diminuição de eficiência laríngea e do controle 
expiratório ${ }^{11,17,21}$. Entretanto, as relações $\mathrm{s} / \mathrm{z}$ e e áfono/e mostraram-se normais para os parkinsonianos, comparando-se aos CC e aos valores esperados para a senilidade ${ }^{12,22}$, sinalizando que ainda há certa coordenação pneumofônica, em discordância das demais medidas como os TMF, a FMR e a IV que se apresentaram alteradas. Deve-se ressaltar que as relações s/z e e áfono/e, resultantes da investigação dos TMF, são parte da avaliação quantitativa da voz ${ }^{17,21,26}$, sendo interpretadas em conjunto, já que TMF /s/ e /z/ alterados podem resultar numa relação $s / z$ matematicamente normal e levar a falsos-negativos ${ }^{26}$.

A restrição da IV, comum no processo de enveIhecimento ${ }^{17,21,27}$ e na DP $^{9-12}$, apresentou-se acentuada nos parkinsonianos em comparação aos CC, que, por sua vez, a apresentaram diminuída em relação à literatura para idosos ${ }^{22}$. A senescência e a DP geram diminuição do controle da atividade motora da musculatura laríngea ${ }^{1,7,12,28}$ e dos músculos respiratórios, dificultando o controle da fonação, além do enrijecimento costal, limitando os movimentos torácicos e as capacidades respiratórias $^{4,5,25,28}$, com diminuição senil da IV, piorada pela DP.

Outra justificativa para tais achados pode ser a hipertonia dos músculos abdominais, devido à manutenção da postura em flexão, tanto nos parkinsonianos quanto nos idosos, com o tronco anteriorizado e com encurtamento desses músculos, prejudicando a FMR ${ }^{6,16,23}$. Ainda, o sexo masculino apresenta maior declínio da $\mathrm{FMR}^{28-30}$, justificando também tais resultados.

Um comentário importante refere-se ao indivíduo de 36 anos com DP, com déficits similares aos outros parkinsonianos em quase todas as medidas, com uma performance negativa nos TMF, IV e na relação e áfono/e possivelmente pelo "parkinsonismo de início precoce", com tendência à sintomatologia mais grave ${ }^{3,24}$.

Entretanto, este parkinsoniano apresentou a Pimáx superior ao seu CC, o que se justificaria pela modificação gradativa da FMR somente a partir das $3^{\text {a }}$ e $4^{\text {a }}$ décadas de vida, mantendo-se estável ou com reduções pouco significativas ${ }^{25,28-30}$, mesmo na presença de DP de início precoce, uma vez que as alterações da DP são assistemáticas ${ }^{1,3,7,10}$.

Existem poucos estudos que relacionam as características clínicas ao início da DP, contudo, quanto mais idoso o parkinsoniano, mais adversa é a evolução da patologia1,3,23. Ainda, a resposta terapêutica individual à levodopa tende a melhorar parâmetros como a IV e a FMR ${ }^{5,10}$ podendo justificar o resultado da Pimáx do parkinsoniano jovem.

Outras explicações advêm de mecanismos que se deterioram à proporção que a idade avança, como o trofismo muscular, o número de unidades motoras, alterações endócrinas, e fatores nutricionais $^{28-30}$. Ainda, pode-se considerar que essas modificações atingem seu pico em torno dos 30 anos, decrescendo progressivamente a partir da $5^{\text {a }}$ década de vida, o que pode justificar o resultado positivo na FMR do parkinsoniano de 36 anos com nível de atividade física ativo.

O CC jovem também apresentou desempenho abaixo do esperado para a faixa etária adulta nas mesmas medidas (TMF, IV e e áfono/e), embora superiores ao CE. Sugere-se que o CC jovem do sexo masculino tenha apresentado um comportamento fonatório ilustrado por hipercinesia na relação e áfono/e, como já mencionado anteriormente ${ }^{17,21}$.

Em relação aos TMF e à IV, que são habilidades dependentes da FMR e cujos valores se mostraram inferiores ao esperado ${ }^{20}$ para o CC jovem, fatores como a quantidade de fibras musculares tipo I e II, nutrição e índice de massa corporal|29,30, podem ter interferido nos resultados abaixo do esperado para o CC jovem.

As alterações posturais típicas da $\mathrm{DP}^{3,23}$ foram mais pronunciadas nos parkinsonianos na faixa entre 50 e 65 anos, supondo-se que a perda de tônus muscular e os afrouxamentos ligamentares, comuns no envelhecimento ${ }^{23,24}$, podem ter modificado as curvaturas vertebrais, ocasionando as alterações encontradas, uma vez que a DP tende a agravar tais distúrbios.

O CE jovem, em comparação com o CC, exibiu menor número de mudanças posturais e o mesmo número de alterações posturais dos demais CC masculinos, o que não era esperado. Uma justificativa decorre do próprio conceito de postura corporal, visto que recebe influências do estado emocional e dos hábitos de vida ${ }^{16,23}$, pois esse sujeito, além de jovem, apresentou nível ativo de atividade física.

Todos os sujeitos tomados como CC apresentaram FMR abaixo do esperado, possivelmente pelo envelhecimento das estruturas, ainda que o desenvolvimento e grau de deterioração dependam de cada indivíduo, por fatores raciais, hereditários, alimentares, sócio-ambientais, e nível de atividade física ${ }^{5,14,20,24,27-30}$.

Ainda, a complexidade neurofisiopatológica da DP, ilustrada por mudanças objetivas ao longo da doença, no mesmo sujeito e em grupos de sujeitos, instiga a realização de estudos prospectivos com amostras maiores que permitem verificar características constantes e comuns, de acordo com as variáveis estudadas, extraindo generalizações e, consequentemente, fornecendo mais subsídios tanto para fins de avaliação quanto de tratamento, uma vez que seus resultados 
possibilitam prever as manifestações da DP, conforme as variáveis envolvidas na doença.

\section{CONCLUSÕES}

No presente estudo, observou-se que, no sexo feminino, o menor estágio da DP e o maior nível de atividade física refletiram-se positivamente sobre as medidas de TMF, a FMR e a relação s/z. A IV mostrou-se rebaixada, porém, mais prejudicada nas parkinsonianas; as alterações posturais foram similares em todos as mulheres.
Os parkinsonianos, independentemente da idade, do estágio da DP e do nível de atividade física, apresentaram prejuízos nos TMF, na IV e na FMR. O parkinsoniano jovem apresentou a Pimáx superior ao seu CC. Os achados de alterações posturais foram maiores nos parkinsonianos de maior idade, independentemente do nível de atividade física.

Verificou-se que todos os CC deste trabalho apresentaram FMR abaixo do previsto para o sexo e a idade, evidenciando que outros fatores podem interferir nesta medida.

\begin{abstract}
Purpose: to check the findings on respiratory muscular strength (RMS), body posture (BP), vocal intensity (VI) and maximum phonation time (MPT), in patients with Parkinson Disease (PD) and control cases, according to gender, Parkinson Disease stage (PD) and the level of physical activity (PA). Methods: three men and two women with PD, between 36 and 63 year old (study cases - SC), and five subjects without neurologic diseases, of the same age, gender and PA level (control cases CC). We evaluated RMS, BP, VI and MPT. Results: men: a more pronounced decrease of MPT, VI, RMS in Parkinson patients, plus postural alterations in the elderly; women: similar postural alterations, positive relation between stages, PA level and the other measures. Conclusions: we observed in women with PD, impaired VI; in men with PD deficits in MPT, VI, RMS. We suggest further studies under an interdisciplinary bias.
\end{abstract}

KEYWORDS: Parkinson's Disease; Respiration; Voice; Posture

\section{REFERÊNCIAS}

1. Ziemssen $T$, Reichmann $H$. Treatment of dysautonomia in extrapyramidal disorders. Therapeutic advances neurol disorders 2010; 3 (1): 53-67.

2. Isaacson $\mathrm{SH}$, Hauser RA. Improving symptom control in early Parkinson's disease.

Therapeutic advances neurol disorders 2009; 2 (6): 393-400.

3. Olanow CW, Stern MB, Sethi K. The scientific and clinical basis for the treatment of Parkinson disease. Neurology 2009; 72:S1-S136.

4. Gross RD, Jr. Atwood CW, Ross SB, Eichhorn KA, Olszewski JW, Doyle PJ. The coordination of breathing and swallowing in Parkinson's Disease. Dysphagia 2008; 23 (2): 136-45.

5. Haas BM, Trew M, Castle PC. Effects of Respiratory Muscle Weakness on Daily Living Function, Quality of Life, Activity Levels and Exercise Capacity in Mild to Moderate Parkinson's disease. American Journal of Physical Medicine \& Rehabilitation 2004; 83: 601-7.
6. Ketelslagers K, De Bodt MS, Wuyts FL, Van de Heyning P. Relevance of subglottic pressure in normal and dysphonic subjects. European Archives of Oto-Rhino-Laryngology 2007; 264 (5): 519-23.

7. Rektorova I, Barrett J, Mikl Michal, Rektor I, Paus $\mathrm{T}$. Functional abnormalities in the primary orofacial sensorimotor cortex during speech in Parkinson's Disease. Movement disorders 2007; 22 (14): 2043-51.

8. Ramig LO, Fox C, Sapir S. Speech treatment for Parkinson's disease. Expert Review of Neurotherapeutics 2008; 8 (2): 297-309.

9. Fox CM, Ramig LO, Ciucci MR, Sapir S, McFarland $\mathrm{DH}$, Farley BG. The science and practice of LSVT/ LOUD: neural plasticity-principled approach to treating individuals with Parkinson disease and other neurological disorders. Seminars in Speech and Language 2006; 27(4): 283-99.

10. Ariatti A, Benuzzi $F$, Nichelli P. Recognition of emotions from visual and prosodic cues in Parkinson's disease. Neurological Sciences 2008; 29 (4): 219-27. 
11. Klostermann F, Ehlen F, Vesper J, Nubel K, Gross M, Marzinzik F, Curio G, Sappok T. Effects of subthalamic deep brain stimulation on dysarthrophonia in Parkinson's disease. J Neurol Neurosurg Psychiatry 2008; 79:522-9.

12. Behlau M. Presbifonia: envelhecimento vocal inerente à idade. In: Russo ICP. Intervenção fonoaudiológica na terceira idade. 1. ed. Rio de Janeiro: Revinter, 1999, cap.3, p.25-50.

13. Feijó $A$, Estrela $F$, Scalco $M$. Avaliação perpectiva e quantitativa da voz na terceira idade. Revista Fonoaudiologia Brasil 1998; 1: 21-9.

14. Benedetti TRB, Antunes PC, Rodriguez-Añez CR, Mazo GZ, Petroski EL. Reprodutibilidade e validade do Questionário Internacional de Atividade Física (IPAQ) em homens idosos. Rev Bras Med Esporte 2007; 13 (1): 11-6.

15. Hoehn MM, Yahr MD. Parkinsonism: onset, progression and mortality. Neurology, 1967;17(5):427-42.

16. Kendall FP, Creary EK. Força Muscular em Relação à Postura - Músculos, provas e funções, São Paulo; Manole, 1995.

17. Pinho SMR. Fundamentos em Fonoaudiologia - Tratando os distúrbios da voz. Rio de Janeiro: Guanabara Koogan S.A., 2003.

18. Koishi HU, Tsuji DH, Imamura R, Sennes LU. Variação da intensidade vocal: estudo da vibração das pregas vocais em seres humanos com videoquimografia. Rev Bras Otorrinolaringol 2003; 69(4): 464-70.

19. Hodge FS, Colton R, Kelley RT. Vocal intensity characteristics in normal and elderly speakers. J Voice 2001; 15(4): 503-11.

20. Neder JA, Andreoni S, Lerarlo MC, Nery LE. Reference values for lung function tests II - Maximal respiratory pressures and voluntary ventilation. Brazilian J Med Biol Res 1999; 32: 719-27.
21. Behlau M, Azevedo R, Pontes P. Conceito de voz normal e classificação das disfonias. In: Behlau, M. Voz: o livro do especialista v.l. Rio de Janeiro: Revinter, 2001, p. 53-84.

22. Colton R, Casper J. Compreendendo os problemas da voz: uma perspectiva fisiológica ao diagnóstico e ao tratamento. Porto Alegre: Artes Médicas; 1996. 386p.

23. Ferreira FV, Prado ALC, Cielo CA, Busanello AR. A relação da postura corporal com a prosódia na Doença de Parkinson: estudo de caso. Rev. CEFAC july/sept. 2007; 9(3): 319-29.

24. Matsudo SM, Gonçalves LHT, Mota JAPS. Uma proposta de política pública de atividade física para idosos. Texto contexto - enfermagem 2007; 16 (3): 387-98.

25. Parreira VF, Guedes LU, Quintão DG, Silveira EP, Tomich GM, Sampaio RF, Britto RR, Goulart F. Padrão respiratório em pacientes portadores da doença de Parkinson e em idosos assintomáticos. Acta Fisiátrica 2003; 10(2): 61-6.

26. Cielo CA, Casarin MT. Sons fricativos surdos. Rev. CEFAC jul/set. 2008; 10(3): 352-8.

27. Huber E, Spruill III J. Age-Related Changes to Speech Breathing With Increased Vocal Loudness J Speech Lang Hear Res june 1 2008; 51(3):651-68.

28. Santos FH, Andrade VM, Bueno OFA. Envelhecimento: um processo multifatorial. Psicologia em estudo 2009; 14 (1): 3-10.

29. Zhong S, Chen CN, Thompson LV. Sarcopenia of ageing: functional, structural and biochemical alterations. Rev. bras. fisioter. 2007; 11 (2): 91-7. 30. Fanò G, Mecocci P, Vecchiet J, Belia S, Fulle S, Polidori C, Felzani G, Senin U, Vecchiet L, Beal F. Age and sex influence on oxidative damage and functional status in human skeletal muscle. J Muscle Res and Cell Motility 2001; 22: 345-51.

http://dx.doi.org/10.1590/S1516-18462010005000103

RECEBIDO EM: 16/01/2010

ACEITO EM: 16/04/2010

Endereço para Correspondência:

Fernanda Vargas Ferreira

Rua Visconde de Pelotas 517, N. Sra. do Rosário

Santa Maria, Rio Grande do Sul, Brasil

CEP: 97010-440

E-mail: nandaf_pg@yahoo.com.br 